\title{
Erratum to: Systematic review of antimicrobials, mucosal coating agents, anesthetics, and analgesics for the management of oral mucositis in cancer patients
}

\author{
Deborah P. Saunders • Joel B. Epstein • Sharon Elad • Justin Allemano • \\ Paolo Bossi • Marianne D. van de Wetering • Nikhil G. Rao • Carin Potting • \\ Karis K. Cheng • Annette Freidank • Michael T. Brennan • Joanne Bowen • \\ Kristopher Dennis - Rajesh V. Lalla $\cdot$ For The Mucositis Study Group of the \\ Multinational Association of Supportive Care in Cancer/International Society \\ of Oral Oncology (MASCC/ISOO)
}

Published online: 5 December 2014

(C) Springer-Verlag Berlin Heidelberg 2014

\section{Erratum to: Support Care Cancer DOI 10.1007/s00520-013-1871-y}

The original version of this paper unfortunately contains errors. Morphine $2 \%$ rinse, should have read as Morphine $0.2 \%$ rinse.

Page 3202, Paragraph 6

The online version of the original article can be found at http://dx.doi.org/ 10.1007/s00520-013-1871-y.

D. P. Saunders $(\bowtie) \cdot$ J. Allemano

Department of Dental Oncology, North East Cancer Center, Health

Sciences North, Sudbury, Ontario, Canada

e-mail: dsaunders@hsnsudbury.ca

\section{J. B. Epstein}

Samuel Oschin Comprehensive Cancer Institute, Cedars Sinai

Medical Center, Los Angeles, CA, USA

J. B. Epstein

City of Hope National Medical Center, Duarte, CA, USA

S. Elad

Division of Oral Medicine, Eastman Institute for Oral Medicine,

University of Rochester Medical Center, Rochester, NY, USA

\section{P. Bossi}

Head and Neck Medical Oncology, Fondazione IRCCS Istituto

Nazionale dei Tumori, Milan, Italy

M. D. van de Wetering

Paediatric Oncology Department, Emma Children's Hospital,

Academic Medical Centre, Amsterdam, The Netherlands

N. G. Rao

Department Radiation Oncology, Moffitt Cancer Center, Tampa, FL, USA
Two studies, including a RCT, examined the use of a $2 \%$ morphine mouth rinse for the management of pain due to $\mathrm{OM}$ in patients receiving chemoradiation for $\mathrm{H} \& \mathrm{~N}$ cancer [21, 22]. Where it should have read as:

Two studies, including a RCT, examined the use of a $0.2 \%$ morphine mouth rinse for the management of pain due to $\mathrm{OM}$ in patients receiving chemoradiation for $\mathrm{H} \& \mathrm{~N}$ cancer [21, 22].

\section{Potting}

Department of Haematology, Radboud University Nijmegen Medical Centre, Nijmegen, The Netherlands

\section{K. K. Cheng}

Alice Lee Centre of Nursing Studies, National University of Singapore, Singapore, Singapore

\section{A. Freidank}

Department of Pharmacy, Klinikum Fulda gAG, Fulda, Germany

M. T. Brennan

Department of Oral Medicine, Carolinas Medical Center, Charlotte, NC, USA

J. Bowen

School of Medical Sciences, University of Adelaide, Adelaide, South Australia, Australia

K. Dennis

Department of Radiation Oncology, Odette Cancer Centre,

Sunnybrook Health Sciences Centre, University of Toronto, Toronto, Ontario, Canada

\section{R. V. Lalla}

Section of Oral Medicine and Neag Comprehensive Cancer Center, University of Connecticut Health Center, Farmington, CT, USA 
Page 3202, Paragraph 7

The panel suggests that morphine $2 \%$ mouth rinse can be effective for the management of pain due to $\mathrm{OM}$ in patients receiving chemoradiation for $\mathrm{H} \& \mathrm{~N}$ cancer (level of evidence III).

Where it should have read as:

The panel suggests that morphine $0.2 \%$ mouth rinse can be effective for the management of pain due to $\mathrm{OM}$ in patients receiving chemoradiation for $\mathrm{H} \& \mathrm{~N}$ cancer (level of evidence III).
Page 3204, Paragraph 3

Although studies with such topical use of opioids were limited, the data did support a suggestion in favor of a $2 \%$ morphine rinse in radiation-induced $\mathrm{OM}$ in $\mathrm{H} \& \mathrm{~N}$ cancer patients.

Where it should have read as:

Although studies with such topical use of opioids were limited, the data did support a suggestion in favor of a $0.2 \%$ morphine rinse in radiation-induced $\mathrm{OM}$ in $\mathrm{H} \& \mathrm{~N}$ cancer patients. 